\title{
Left Atrial Appendage Occlusion Device: Evaluation of Surgical Implant Success and in Vivo Corrosion Performance
}

\author{
Jonathan Snyder ${ }^{1}$, Amy M. Engel ${ }^{2}$, Kevin C. White ${ }^{3}$, Noah Budiansky ${ }^{3}$, \\ J. Michael Smith ${ }^{1}$ \\ ${ }^{1}$ Department of Surgery, Good Samaritan Hospital, Cincinnati, USA \\ ${ }^{2}$ E. Kenneth Hatton MD Institute for Research and Education, Cincinnati, USA \\ ${ }^{3}$ Exponent Inc., Natick, Massachusetts, USA \\ Email: amy_engel@trihealth.com
}

Received September 21, 2011; revised November 26, 2011; accepted December 3, 2011

\begin{abstract}
Objective: The purpose of this study was to compare the in vivo corrosion resistance of the implanted titanium, nitinol annular occlusion device to a control device, i.e. an implantable device containing nitinol, approved by the FDA and currently on the market. Methods: The annular occlusion device is a self-closing, implantable clip. Three canines underwent placement of devices on the left and right atrial appendages. Two Vnus U-clips were secured to either atrium. On post-operative day 95, animals underwent en-bloc cardiac resection via the previous left thoracotomy incision. The annular occlusion device and U-clips were dissected free from the atria. The polyester fabric and tissue ingrowth were removed from the devices and were sent for corrosion analysis. Results: Gross examination of resected hearts of two canines revealed no abnormalities. The compressed endocardial surfaces were completely fused and the appendages fully necrosed. All devices were located and harvested. The annular occlusion device clips and Medtronic Vnus U-clips were evaluated using scanning electron microscopy. Both low and high magnification examination of the nitinol springs and the site of insertion of the nitinol springs into the titanium tubes in the annular occlusion device showed no evidence of localized corrosion. In no case was any evidence of general or localized corrosion found in the form of metallic oxidation. Conclusion: The annular occlusion device provides safe and reliable exclusion of the left atrial appendage without evidence of general or localized corrosion over the 95-day exposure period in canines and may therefore provide a reasonable therapeutic option for stroke risk reduction in patients with atrial fibrillation.
\end{abstract}

Keywords: Atrial Appendage Occlusion Device; Atrial Fibrillation; Exclusion of Left Atrial Appendage

\section{Introduction}

Atrial fibrillation (AF) is a well-known cardiac arrhythmia and is associated with significant morbidity and mortality, often from thromboembolic events originating in the left atrial appendage (LAA) [1]. Patients with AF have a five-fold increase of stroke risk, and it has been reported that $60 \%-90 \%$ of left atrial thrombi originate in the LAA [2,3]. Relative stagnation of blood in the atrium during AF promotes local coagulation and generation of thrombi. Historically, the gold standard of treatment for AF has been oral anticoagulation therapy [4,5]. Given the morbidity associated with this type of therapy, as well as difficulties with compliance and appropriate monitoring, a shifting focus in recent years has been towards surgical exclusion of the LAA in patients with atrial fibrillation undergoing cardiac surgery [6-10]. In 2009, the Project AF trial demonstrated non-inferiority of percutaneous closure of the LAA when compared to oral anticoagulation therapy for stroke prevention in patients with atrial fibrillation [11].

Recent technological advances have allowed for complete exclusion of the LAA [12]. Several devices have been developed to allow for an epicardial approach, which precludes the need for transseptal puncture, or left endocardial catheters with their associated risk of embolic events and eliminates the technical limitations and complications of stapling technique and the cut-and-sew method.

A new clip used for LAA occlusion in patients with AF undergoing cardiac surgery has been evaluated and determined to provide safe, rapid and reliable occlusion of the LAA in both canine model and clinical trials [1316]. Histologic evaluation has been performed at multiple survival durations, up to 180 days. The corrosion 
resistance of the Annular Occlusion Device (AOD) has been investigated in vitro in accordance with American Society for Testing and Materials (ASTM) standard F2129-06 [17]. This standard also calls out that in vitro corrosion parameters cannot be reasonably assumed to correlate with in vivo conditions. The standard also recommends that a similar reference device with a similar purpose be tested under the same conditions. The purpose of this study is to evaluate and compare the in vivo corrosion resistance of the implanted (AOD) against a control device that has been FDA approved and is in use in the medical community today.

\section{Materials and Methods}

\subsection{Device Description}

The AOD was developed by AtriCure, Inc. (Cincinnati, Ohio, USA) and is a self-closing, implantable clip. A reusable deployment tool was used in this trial which allows for placement and, if necessary, repositioning before deployment. A sizing tool provided with the clip aids in selecting the appropriate size clip. The clip is composed of two parallel rigid titanium tubes joined at both ends by nitinol springs. The springs are constructed from electropolished and passivated nitinol. The titanium tubes are encased in a urethane elastomer and the entire device is wrapped in a knit braided polyester. The polyester fabric, promotes tissue ingrowth, and prevents trauma to or erosion into adjacent anatomic structures, such as the left ventricle or pulmonary artery. During deployment, the AOD is placed over the appendage and positioned at the base. Once properly positioned, the AOD is deployed and closes at a specific pressure, bringing the two walls of the appendage together which atraumatically occludes the structure. Over time, the appendage walls fuse together, the appendage necroses, tissue ingrowth into the knit braid keeps the device in place, and the endocardial seam is smoothed over by a layer epithelial tissue.

\subsection{Animal Model}

Three mongrel canines (mean weight $27.7 \mathrm{~kg}+/-1.8 \mathrm{~kg}$ ) were used in the study. Their use was approved by Good Samaritan Hospital's Institutional Animal Care and Use Committee. They received humane treatment under the supervision of an animal lab supervisor and technician, in accordance with the "Guide for the Care and Use of Laboratory Animals” prepared by the Institute of Laboratory Resources National Research Council, published by the National Academy Press, revised 1996.

\subsection{Implantation and Device Harvest}

All animals underwent general endotracheal anesthesia, followed by left thoracotomy and pericardial incision. After appropriate sizing, $35 \mathrm{~mm}$ AODs were deployed on the left and right atrial appendages, with two devices used per animal (Figure 1). Two Vnus U-clips were secured to either atrium, with a total of four U-clips implanted per animal. The thoracotomies were closed with no intra-operative surgical complications in any of the three animals. The animals did not receive systemic anticoagulation or anti-platelet therapy during or after the procedure.

The canines were recovered in standard housing where they underwent routine monitoring and assessment to determine progression of healing and adequacy of analgesia. Prior to initiation of the study, the survival period was determined to be no less than ninety days. On postoperative day 95, the animals underwent general anesthesia, humane euthanasia and en-bloc cardiac resection via the previous left thoracotomy incision. Photographs of the devices were taken during the implantation and harvest.

The AODs and U-clips were sharply dissected free from the atria, with care being taken not to contact any device directly with a metallic instrument. The polyester fabric and tissue ingrowth were removed from the devices, which were immediately placed in mineral oil to suspend their state of corrosion. The preserved samples were shipped to a third-party consulting firm Exponent, Inc (Natick, MA, USA) for corrosion analysis.

\subsection{Corrosion Analysis}

Upon receipt by Exponent, Inc., the mineral oil was removed by ultrasonic cleaning in hexane. In order to remove retained biologic material, the devices were ultra-

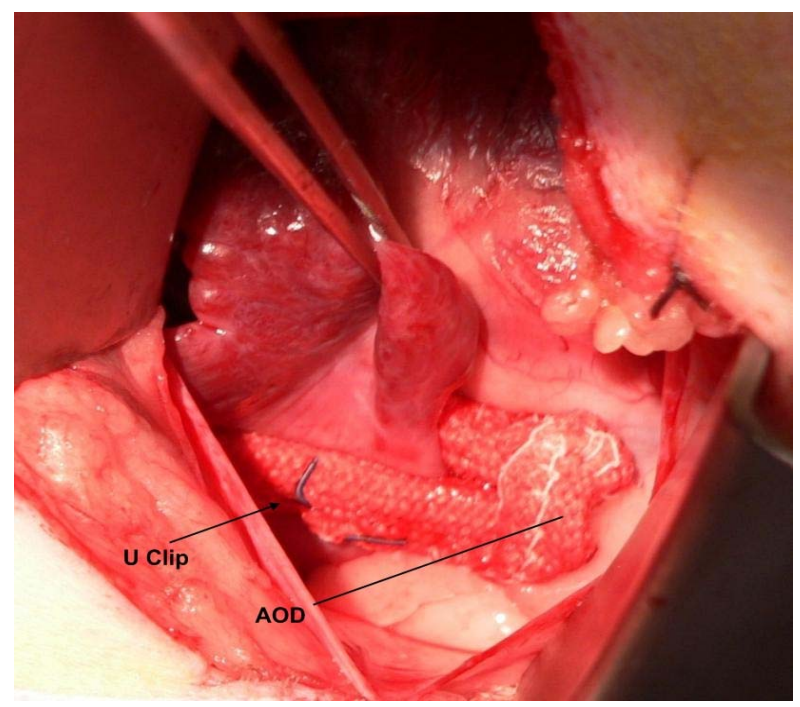

Figure 1. Implantation photograph of annular occlusion device (AOD) deployed at the base of the left atrial appendage in a living canine. 
sonically cleaned in ethanol, then with alkaline laboratory detergent and a nylon brush to avoid causing mechanical damage to the metallic surfaces. The samples were then rinsed in water, ultrasonically cleaned in ethanol and allowed to dry prior to inspection using scanning electron microscopy (SEM) (JEOL Model 6390LV). SEM images were collected at high vacuum in secondary electron imaging mode to enhance surface topography. Energy dispersive x-ray spectroscopy (EDS) was used to determine the chemical elements present on the surface of the samples. Images were taken at selected and representative areas under high magnification where necessary.

\section{Results}

\subsection{Summary}

All implantation and harvest surgeries were carried out without incident. On implantation, AOD placement was performed simply and without injury to surrounding structures. In the first animal, the AODs were placed at the base of the atrial appendages requiring the U-clips to be placed in a position contacting the fabric of the AOD. In the subsequent animals, the AOD was not advanced to the base of the appendage to accommodate U-clip placement without contact. All six appendages were sized with the provided tool and the smallest AOD (35 mm) was implanted in all cases. Tissue engagement of $40 \%-75 \%$ of the length of the device was noted. The compressed appendage thickness was $1 \mathrm{~mm}$ in all six devices.

The canines were survived for 95 days. Upon harvest, gross examination of resected hearts of canines one and two revealed no abnormalities-the compressed endocardial surfaces were completely fused and the appendages fully necrosed (Figure 2). All devices were located and harvested. On post-operative day 35, canine number three was examined by the staff veterinarian due to decreased activity and appetite and was administered an antibiotic for a suspected infection. The animal recovered fully, but did not regain lost weight. The occluded portion of the right atrial appendage of canine three was not fully necrosed and upon being cut open contained coagulated blood. The endocardial surfaces of both atrial lumens were fully closed. Gross examination of the pericardium and ventricles of canine number three revealed evidence of epicardial inflammation. This observation suggests that an impaired healing process may have prevented full necrosis of the right atrial appendage. Full occlusion was confirmed following AOD implant and verified at explant for all six appendages.

\subsection{Corrosion Analysis}

AOD clips and Medtronic Vnus U-clips were analyzed using SEM. Low magnification examination of the AOD nitinol springs and the site of insertion of the nitinol springs into the titanium tubes showed no evidence of general or localized corrosion. In many cases, surface staining was observed, but no indications of gross metal dissolution such as would be associated with general corrosion or localized corrosion were observed. All implant devices will have a detectible but quite low metal dissolution rate associated with the passive film. The sites of nitinol spring insertion into the titanium tubes often revealed retained biologic material (Figure 3). In addition, striation marks were seen along the entire length of the nitinol wires and are consistent with surface morphology expected from the manufacturing process.

EDS indicated that clean nitinol surfaces were composed of titanium, nickel, oxygen, and trace amounts of carbon. The source of carbon is unknown, but likely

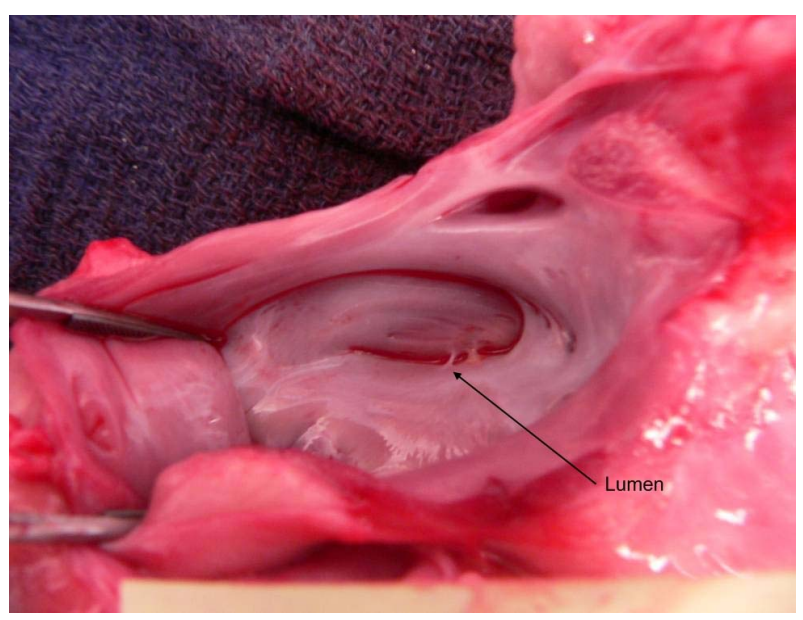

Figure 2. Explantation photograph looking into opened left atrium with endocardial fusion of fully occluded appendage.

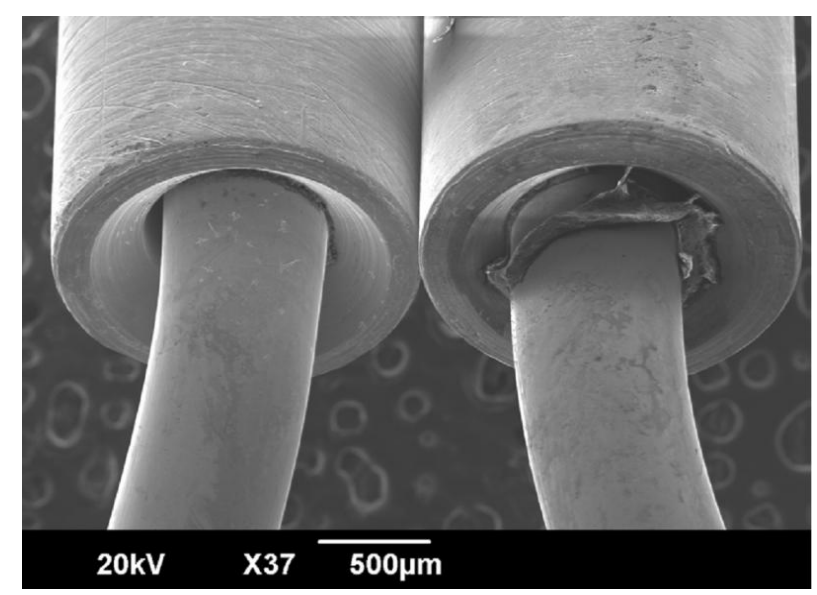

Figure 3. Evaluation of the region where the nitinol springs inserted into the titanium rods reveals no corrosion damage. Note the retained biologic material on the sample at the site of insertion. 
comes either from carbon in the alloy itself or carbon which is present on the surface of the device. The darkened regions were found to have a high concentration of carbon that was consistent with organic material present on the surfaces of the samples. Assuming that the nitinol material is homogeneous these regions were attributed to the presence of biologic material or retained mineral oil from shipping.

Similar SEM and EDS analysis of the Medtronic Vnus U-clips did not show any evidence of general or localized corrosion (Figure 4). One area identified on a Vnus U-clip showed characteristics which could be associated with incipient corrosion but it was not possible to rule out surface damage induced during to the U-clip manufacturing process (Figure 5).

\section{Discussion}

Atrial fibrillation (AF) is the most common cardiac arrhythmia and is a source of secondary morbidity in the form of cardiomyopathy, congestive heart failure and generation of thromboembolic events [1]. AF increases in prevalence with increasing age, which makes the adherence to the gold standard of oral anticoagulation difficult from the standpoint of compliance and management of bleeding complications [4,5]. The left atrial appendage (LAA) is believed to be the source of thrombus in over $90 \%$ of nonrheumatic patients with atrial fibrillation $[2,3]$.

With advances in cardiothoracic surgery in recent years, there has been a paradigm shift from purely medical management of atrial fibrillation to mechanical and surgical exclusion of the LAA as a means of reducing the risk of thrombus generation. Pioneers of this pursuit who began with stapling techniques and "cut and sew” method of atrial exclusion have met with often incomplete results and significant complications of secondary rupture and large "cul-de-sac" formation [18]. With the development of the epicardial AOD, occlusion of the LAA on a beating heart has been made feasible without the need for transseptal perforation and with relative ease and reproducibility of deployment. Previous studies have validated its ability to electrically isolate and generate necrosis in the LAA, while promoting growth of a smooth epicardial surface at the site of occlusion and maintaining fixation due to its knit braided polyester sheath [12-16].

This study showed a lack of pitting corrosion or significant general corrosion in implanted AODs after 95 days of in vivo implantation in a canine host. The implanted devices were removed after gross examination and smooth, endothelial closure of the atrial surface of the LAA was confirmed. The explanted devices were examined using SEM. A comprehensive SEM examination of the explanted devices failed to find any evidence

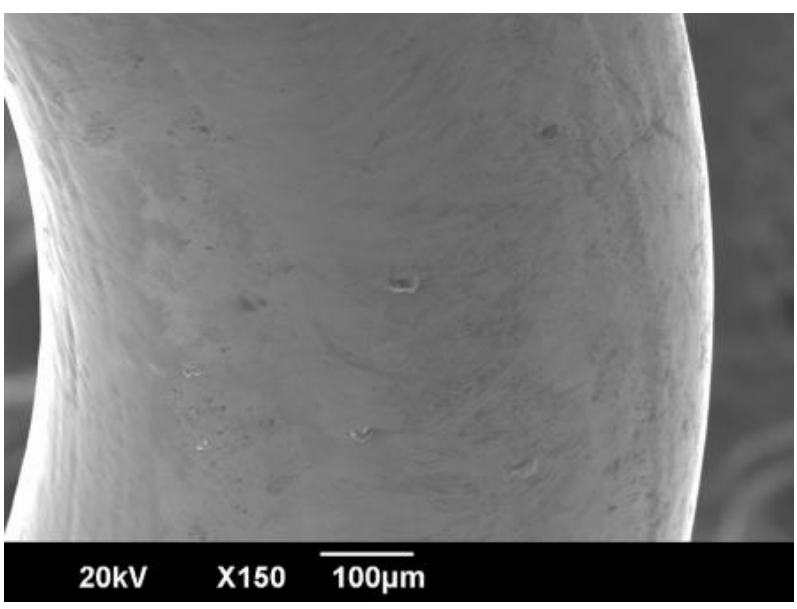

Figure 4. High magnification image of AOD nitinol spring, showing surface texture and debris but no evidence of corrosion.

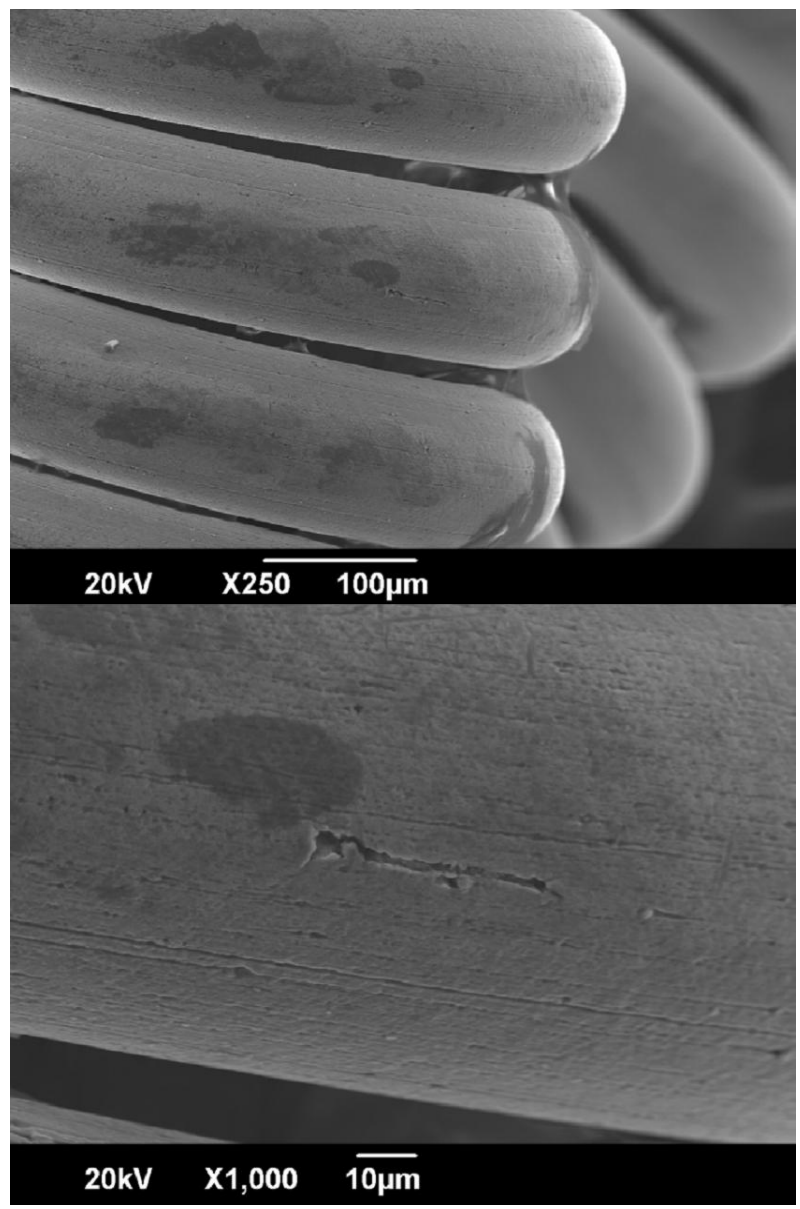

Figure 5. SEM image of Vnus U-clip after 90 day implantation. High magnification image demonstrates possible corrosion versus damage from wire drawing.

of localized corrosion on the titanium tubes or the attached nitinol springs. The reference control for this study was the Medtronic Vnus U-clip, which is released 
into circulation and approved for use in cardiovascular surgery. The control implants also showed no clear evidence of general or localized corrosion.

This study is limited by its size and relatively brief follow-up duration of 95 days. The 95-days implant time period is too brief a time period to draw any conclusions regarding corrosion-fatigue effects. Also, implantation and harvest for examination using SEM is not a feasible option for clinical trials, so our understanding of corrosion in humans may be limited to animal or perhaps someday cadaveric models. Accordingly, data must be extrapolated from the canine model to the human model under the assumption that substantially similar biochemical and mechanical factors exist to induce any potential disruption in the surface and integrity of synthetic, metallic implants.

The Annular Occlusion Device provides safe and reliable exclusion of the LAA without evidence of general or localized corrosion during a 95-day implant period and therefore may provide a reasonable therapeutic option for stroke risk reduction in patients with AF.

\section{Acknowledgements}

Funding for this project was provided by ArtiCure (Cincinnati, Ohio, USA).

\section{REFERENCES}

[1] P. A. Wolf, R. D. Abbott and W. B. Kannel, “Atrial Fibrillation as an Independent Risk Factor for Stroke: The Framingham Study,” Stroke, Vol. 22, No. 8, 1991, pp. 983-988. doi:10.1161/01.STR.22.8.983

[2] J. L. Blackshear and J. A. Odell, “Appendage Obliteration to Reduce Stroke in Cardiac Surgical Patients with Atrial Fibrillation,” Annals of Thoracic Surgery, Vol. 61, No. 2, 1996, pp. 755-759. doi:10.1016/0003-4975(95)00887-X

[3] N. M. Al-Saady, O. A. Obel and A. J. Camm, "Left Atrial Appendage: Structure, Function, and Role in Thromboembolism,” Heart, Vol. 82, No. 5, 1999, pp. 547-554.

[4] A. M. Lee, S. G. Melby and R. J. Damiano Jr., "The Surgical Treatment of Atrial Fibrillation," Surgery Clinics of North America, Vol. 89, No. 4, 2009, pp. 1001-1020. doi:10.1016/j.suc.2009.06.001

[5] V. Fuster, L. E. Rydén, D. S. Cannom, H. J. Crijns, A. B. Curtis, K. A. Ellenbogen, et al., “ACC/AHA/ESC 2006 Guidelines for the Management of Patients With Atrial Fibrillation-Executive Summary: A Report of the American College of Cardiology/American Heart Association Task Force on Pactice Guidelines and the European Society of Cardiology Committee for Practice Guidelines (Writing Committee to Revise the 2001 Guidelines for the Management of Patients with Atrial Fibrillation) Developed in Collaboration with the European Heart Rhythm Association and the Heart Rhythm Society,” Journal of the American College of Cardiology, Vol. 48, 2006, pp. 854-906. doi:10.1016/j.jacc.2006.07.009
[6] T. Nakai, M. D. Lesh, E. P. Gerstenfeld, R. Virmani, R. Jones and R. J. Lee, "Percutaneous Left Atrial Appendage Occlusion (PLAATO) for Preventing Cardiuembolism: First Experience in a Canine Model,” Circulation, Vol. 105, No. 18, 2002, pp. 2217-2222. doi:10.1161/01.CIR.0000015605.30810.51

[7] I. R. Hanna, P. Kolm, R. Martin, M. Reisman, W. Gray and P. C. Block, "Left Atrial Structure and Function after Percutaneous Left Atrial Appendage Transcatheter Occlusion (PLAATO): Six-Month Echocardiographic Follow-Up," Journal of the American College of Cardiology, Vol. 43, No. 10, 2004, pp. 1868-1872. doi:10.1016/j.jacc.2003.12.050

[8] H. Sievert, M. D. Lesh, T. Trepels, H. Omran, A. Bartorelli, P. D. Bella, et al., "Percutaneous Left Atrial Appendage Transcatheter Occlusion to Prevent Stroke in High-Risk Patients with Atrial Fibrillation: Early Clinical Experience,” Circulation, Vol. 105, 2002, pp. 1868-1872. doi:10.1161/01.CIR.0000015698.54752.6D

[9] B. Meier, I. Palacios, S. Windecker, M. Rotter, Q. L. Cao, D. Keane, et al., "Transcatheter Left Atrial Appendage Occlusion with Amplatzer Devices to Obviate Anticoagulation in Patients with Atrial Fibrillation," Catheterization and Cardiovascular, Vol. 60, No. 3, 2003, pp. 417-422. doi:10.1002/ccd.10660

[10] J. A. Odell, J. L. Blackshear, E. Davies, W. J. Byrne, C. F. Kollmorgen, W. D. Edward, et al., "Thoracoscopic Obliteration of the Left Atrial Appendage: Potential for Stroke Reduction?” Annals of Thoracic Surgery, Vol. 61, No. 2, 1996, pp. 565-569.

doi:10.1016/0003-4975(95)00885-3

[11] D. R. Holmes, V. Y. Reddy, Z. G. Turi, S. K. Doshi, H. Sievert, M. Buchbinder, C. M. Mullin and P. Sick, PROJECT AF Investigators, "Percutaneous Closure of the Left Atrial Appendage versus Warfarin Therapy for Prevention of Stroke in Patients with Atrial Fibrillation: And Randomised Non-Inferiority Trial,” Lancet, Vol. 374, No. 9689, 2009, pp. 534-542. doi:10.1016/S0140-6736(09)61343-X

[12] K. Kamohara, K. Fukamachi, Y. Ootaki, M. Akiyama, F. Zahr, M. W. Kopcak Jr., et al., "A Novel Device for Left Atrial Appendage Exclusion,” Journal of Cardiovascular Surgery, Vol. 130, No. 6, 2005, pp. 1639-1644. doi:10.1016/j.jtcvs.2005.08.019

[13] K. Kamohara, K. Fukamachi, Y. Ootaki, M. Akiyama, F. Cingoz, C. Ootaki, et al., "Evaluation of a Novel Device for Left Atrial Appendage Exclusion: The Second-Generation Atrial Exclusion Device,” Journal of Cardiovascular Surgery, Vol. 132, No. 2, 2006, pp. 340-346. doi:10.1016/j.jtcvs.2006.04.021

[14] S. P. Salzberg, A. Plass, M. Y. Emmert, L. Desbiolles, H. Alkadhi, J. Grünenfelder and M. Genomi, "Left Atrial Appendage Clip Occlusion: Early Clinical Results,” Journal of Cardiovascular Surgery, Vol. 139, No. 5, 2010, pp. 1269-1274. doi:10.1016/j.jtcvs.2009.06.033

[15] H. Fumoto, A. M. Gillinov, Y. Ootaki, M. Akiyama, D. Saeed, T. Horai, C. Ootaki, et al., “A Novel Device for Left Atrial Appendage Exclusion: The Third-Generation Atrial Exclusion Device,” Journal of Thoracic and Car- 
diovascular Surgery, Vol. 136, No. 4, 2008, pp. 10191027. doi:10.1016/j.jtcvs.2008.06.002

[16] S. P. Salzberg, A. M. Gillinov, A. Anyanwu, J. Castillo, F. Filsoufi and D. H. Adams, "Surgical Left Atrial Appendage Occlusion: Evaluation of a Novel Device with Magnetic Resonance Imaging," European Journal of CardioThoracic Surgery, Vol. 34, No. 4, 2008, pp. 766-770.

[17] Annual Book of ASTM Standards, “ASTM F2129-06: Standard Test Method for Conducting Cyclic Potentiodynamic Polarization Measurements to Determine the
Corrosion Susceptibility of Small Implant Devices," ASTM International, West Conshohocken, 2006.

[18] S. H. Ostermayer, M. Reisman, P. H. Kramer, R. V. Matthews, W. A. Gray, P. C. Block, H. Omran, et al., "Percutaneous Left Atrial Appendage Transcatheter Occlusion (PLAATO System) to Prevent Stroke in High-Risk Patients with Non-Rheumatic Atrial Fibrillation: Results from the International Multi-Center Feasibility Trials,” Journal of the American College of Cardiology, Vol. 46, No. 1, 2005, pp. 9-14. doi:10.1016/j.jacc.2005.03.042 